\title{
On the Description of Vector Bosons
}

\author{
Konstantin Karplyuk
}

\begin{abstract}
Department of Radiophysics, Taras Shevchenko University, Academic Glushkov prospect 2, building 5, Kyiv 03122, Ukraine
\end{abstract}
Corresponding Author: karpks@hotmail.com

Copyright (C)2013 Horizon Research Publishing All rights reserved.

\begin{abstract}
An alternative description to the Weinberg-Salam model of charged currents interaction is proposed. The description does not use local calibration symmetry and spontaneous symmetry breaking. The description is based on the vector boson representation governed by the modified Proca equations. The proposed equations describe processes with non-conserved charges. This modification changes the boson propagator, thus the naive theory with the vector-boson interaction becomes a renormalized one. This approach to the description of the charged current interaction leads to the same Lagrangian as the Weinberg-Salam model.
\end{abstract}

Keywords Vector bosons, Charged Current Interaction, Renormalized theory

\section{Introduction}

It is known that massive $\mathrm{W}$ - and Z- boson exchange is responsible for the weak interaction. The WeinbergSalam [1, 2] model uses local calibration symmetry and spontaneous symmetry breaking for their description. In this model gauged bosons of the $\mathrm{SU}(2)$ group described by nonlinear equations, but no nonlinear behavior in $\mathrm{W}$ - and Z- boson discovered yet. Besides, in order to assign mass to the massless gauged bosons the Higgs bosons are used. That is why it is reasonable to look for another alternative to the intermediate massive boson description.

The justification of the necessity of local calibration symmetry and spontaneous symmetry breaking is the non-renormalizability of the naive theory with the intermediate massive bosons. The Proca equation is usually used for the description of massive vector bosons:

$$
\partial_{\mu} F^{\mu \nu}+\varkappa^{2} X^{\nu}=\mathfrak{j}_{e}^{\nu}
$$

Where, $\varkappa=m c / \hbar$,

$$
F^{\mu \nu}=\partial^{\mu} X^{\nu}-\partial^{\nu} X^{\mu}
$$

$X^{\nu}$ is the vector potential, which is determined by the equation

$$
\partial_{\alpha} \partial^{\alpha} X^{\nu}+\varkappa^{2} X^{\nu}=\mathfrak{j}_{e}^{\nu}+\frac{1}{\varkappa^{2}} \partial^{\nu} \partial_{\mu} \mathfrak{j}_{e}^{\mu}
$$

However, Eqs. (1)-(3) lead to unacceptable results. The propagator of the boson described by Eq. (1) has the form

$$
D_{\mu \nu}=-\frac{\eta_{\mu \nu}}{k^{2}-\varkappa^{2}}+\frac{1}{k^{2}-\varkappa^{2}} \frac{k_{\mu} k_{\nu}}{\varkappa^{2}},
$$

where $\eta_{\mu \nu}=\operatorname{diag}(1,-1,-1,-1)$, and $k_{\mu}$ is the wave vector. The second term on the right-hand side of Eq. (4) is the offending term and makes the theory with the boson interaction a non-renormalized one. To save renormalization, in the Weinberg-Salam electroweak model the contribution from the second term is compensated for by the contribution from the hypothetical Higgs bosons.

It should be noted that the second term in Eq. (4) is associated with the non-conserved current in Eq. (3). If the current is conserved, $\partial_{\mu} \mathrm{j}_{e}^{\mu}=0$, and this term is absent.

\section{Methodology}

We propose another way to eliminate the second term in Eq. (4). To describe the vector bosons let us use the equation

$$
\partial^{\nu} \epsilon+\partial_{\mu} F^{\mu \nu}+\varkappa^{2} X^{\nu}=\mathfrak{j}_{e}^{\nu}
$$

instead of the Proca equation. Where the scalar field $\epsilon$ is given by:

$$
\epsilon=\partial_{\alpha} X^{\alpha}
$$

In this case the potential is given by the equation

$$
\partial_{\alpha} \partial^{\alpha} X^{\nu}+\varkappa^{2} X^{\nu}=\square X^{\nu}+\varkappa^{2} X^{\nu}=\mathfrak{j}_{e}^{\nu}
$$

where $\square=c^{-2} \partial^{2} / \partial t^{2}-\Delta$ is the d'Alambertian. The propagator of the boson described by equations (5), and (7) has the form

$$
D_{\mu \nu}=-\frac{\eta_{\mu \nu}}{k^{2}-\varkappa^{2}}
$$

Thus, the introduction of the scalar field $\epsilon$ improves the propagator (4). The propagator (8) is only different from the photon one by the mass in the denominator. It allows continuous transition to the massless boson, and coincides with the photon propagator when $k \rightarrow \infty$. The most important consequence is that the theory based on such propagator becomes renormalized. 
The necessity of introduction of the scalar field $\epsilon$ is associated with a non-conserved current, but not associated with the mass of the bosons. Differentiation of Eq. (5) yields the equation for the scalar field $\epsilon$ :

$$
\partial_{\nu} \partial^{\nu} \epsilon+\varkappa^{2} \epsilon=\partial_{\nu} \dot{j}_{e}^{\nu}
$$

As one can see the scalar field $\epsilon$ is generated only by a non-conserved current, which does not satisfy the equation of continuity

$$
\partial_{\nu} \dot{j}_{e}^{\nu}=0
$$

We must note that should the electric charge not be conserved, it will also be necessary to change Maxwell's equations in a similar way.

The above consideration of the vector boson description can be extended to the pseudovector bosons. To describe them we propose to use the equation

$$
\partial^{\kappa} \beta+\frac{1}{2} \varepsilon^{\kappa \lambda \mu \nu} \partial_{\lambda} F_{\mu \nu}+\varkappa^{2} Y^{\kappa}=\mathfrak{j}_{m}^{\kappa}
$$

where

$$
\beta=\partial_{\nu} Y^{\nu}, \quad F^{\kappa \lambda}=\varepsilon^{\kappa \lambda \mu \nu} \partial_{\mu} Y_{\nu}
$$

Potential $Y^{\nu}$ is given by the same equation as potential $X^{\nu}$ :

$$
\partial_{\alpha} \partial^{\alpha} Y^{\nu}+\varkappa^{2} Y^{\nu}=\square Y^{\nu}+\varkappa^{2} Y^{\nu}=\mathfrak{j}_{m}^{\nu}
$$

Bosons described by (7) and (13) we call vector and pseudo-vector bosons because they are described by 4 - vector and 4 - pseudovector potentials. There are no constraints on these potentials, that is why these bosons have four internal degrees of freedom each. It's mean that they are multi-spinal and can exist in two states: with spine 1 and spine 0 . Properties of such bosons were discussed by Kruglov [3, 4, 5, 6]. Conclusion about interaction of non-conserved currents via multi-spine bosons can be checked experimentally.

Description of vector and pseudo-vector bosons with the same mass can be combined. It is useful to do, because the weak interaction is just $V-A$ character. We can introduce such a joint description if we associate the field $F^{\mu \nu}$ with the two potentials $X^{\nu}$ and $Y^{\nu}$ simultaneously,

$$
F^{\kappa \lambda}=\partial^{\kappa} X^{\lambda}-\partial^{\lambda} X^{\kappa}+\varepsilon^{\kappa \lambda \mu \nu} \partial_{\mu} Y_{\nu}
$$

and use both the Eqs. (5) and (11). We write down these equations in three-dimensional form:

$$
\begin{aligned}
\frac{1}{c} \frac{\partial \epsilon}{\partial t}+\nabla \cdot \boldsymbol{E}-\varkappa V^{0} & =\zeta c \rho_{e}, \\
\frac{1}{c} \frac{\partial \boldsymbol{E}}{\partial t}-\nabla \times \mathfrak{B}+\nabla \epsilon+\varkappa \boldsymbol{V} & =-\zeta \boldsymbol{j}_{e}, \\
-\frac{1}{c} \frac{\partial \beta}{\partial t}+\nabla \cdot \mathfrak{B}+\varkappa U^{0} & =-\zeta c \rho_{m}, \\
\frac{1}{c} \frac{\partial \mathfrak{B}}{\partial t}+\nabla \times \boldsymbol{E}-\nabla \beta-\varkappa \boldsymbol{U} & =\zeta \boldsymbol{j}_{m} .
\end{aligned}
$$

Here,

$$
\begin{aligned}
& \boldsymbol{E}=-\frac{1}{c} \frac{\partial \boldsymbol{X}}{\partial t}-\nabla X^{0}+\nabla \times \boldsymbol{Y}, \\
& \boldsymbol{B}=\frac{1}{c} \frac{\partial \boldsymbol{Y}}{\partial t}+\nabla Y^{0}+\nabla \times \boldsymbol{X}, \\
& \epsilon=\frac{1}{c} \frac{\partial X^{0}}{\partial t}+\nabla \cdot \boldsymbol{X}, \quad V^{0}=-\varkappa X^{0} \\
& \boldsymbol{V}=-\varkappa \boldsymbol{X} \\
& \beta=\frac{1}{c} \frac{\partial Y^{0}}{\partial t}+\nabla \cdot \boldsymbol{Y}, U^{0}=-\varkappa Y^{0}, \\
& \boldsymbol{U}=-\varkappa \boldsymbol{Y} .
\end{aligned}
$$

The three-dimensional coordinates of the vectors and tensors are given by the rules: $\boldsymbol{E}=\left(F_{01}, F_{02}, F_{03}\right)$, $\mathfrak{B}=\left(-F_{23},-F_{31},-F_{12}\right), \boldsymbol{X}=\left(X^{1}, X^{2}, X^{3}\right), \boldsymbol{Y}=$ $\left(Y^{1}, Y^{2}, Y^{3}\right), \zeta c \rho_{e}=\zeta j_{e}^{0}=\mathfrak{j}_{e}^{0}, \zeta j_{e}=\left(\mathfrak{j}_{e}^{1}, \mathfrak{j}_{e}^{2}, \mathfrak{j}_{e}^{3}\right), \zeta c \rho_{m}=$ $\zeta j_{m}^{0}=\mathfrak{j}_{m}^{0}, \zeta \boldsymbol{j}_{m}=\left(\mathfrak{j}_{m}^{1}, \mathfrak{j}_{m}^{2}, \mathfrak{j}_{m}^{3}\right)$. The coefficients $c=$ $1 / \sqrt{\varepsilon_{0} \mu_{0}}$ and $\zeta=\sqrt{\frac{\mu_{0}}{\varepsilon_{0}}}$ are introduced in the definitions of the three-dimensional variables so that in case the $\varkappa=0, j_{m}^{\mu}=0, Y^{\mu}=0, \epsilon=0$, Eqs. (15)-(18) coincide with Maxwell's equations, written down in SI unit system. In this case we postulate $\mathfrak{B}=c \boldsymbol{B}, X^{0}=\varphi$, $\boldsymbol{X}=c \boldsymbol{A}$ and use the constants $c$ and $\zeta$ instead of the constants $\varepsilon_{0}$ and $\mu_{0}$. This form of Maxwell's equations allows us to use all the advantages of the Gaussian unit system, remaining at the same time in the SI unit system.

Note that electromagnetic field definition via two potentials (vector potential and pseudo-vector potential) was used before for the description of the hypothetical magnetic monopole fields [7].

The following equalities

$$
\begin{aligned}
\frac{1}{c} \frac{\partial V^{0}}{\partial t}+\nabla \cdot \boldsymbol{V}+\varkappa \epsilon & =0, \\
\frac{1}{c} \frac{\partial \boldsymbol{V}}{\partial t}-\nabla \times \boldsymbol{U}+\nabla V^{0}-\varkappa \boldsymbol{E} & =0, \\
\frac{1}{c} \frac{\partial \boldsymbol{U}}{\partial t}+\nabla \times \boldsymbol{V}+\nabla U^{0}+\varkappa \mathfrak{B} & =0, \\
\frac{1}{c} \frac{\partial U^{0}}{\partial t}+\nabla \cdot \boldsymbol{U}+\varkappa \beta & =0,
\end{aligned}
$$

are satisfied due to the definitions (19)-(22). It may seem strange to introduce the fields $V^{\mu}$ and $U^{\mu}$, which are different from the potentials only by factor $\varkappa$. However, Eqs. (15)-(18) and (23)-(26) allow the introduction of the tensor, scalar and pseudo-scalar potentials also. The fields $V^{\mu}$ and $U^{\mu}$ are connected with such potentials by differential relations like (19)-(20), and the fields $\epsilon, \beta$, $\boldsymbol{E}, \mathfrak{B}$ by the multiplier $\varkappa$. So, the fields $V^{\mu}, U^{\mu}$ are connected with all potentials in the same way as the fields $\epsilon, \beta, \boldsymbol{E}, \mathfrak{B}$.

Substitution of the expressions (19)-(22) into Eqs. (15)-(18) yields equations (7) and (13) for the potentials.

The Eqs. (15)-(18) are the Euler equations for the Lagrangian

$$
\begin{gathered}
L=L_{b}+L_{i}, \\
L_{b}=\frac{1}{2 \zeta}\left(-\frac{1}{2} F_{\alpha \beta} F^{\alpha \beta}-\epsilon^{2}+\beta^{2}+V_{\alpha} V^{\alpha}-U_{\alpha} U^{\alpha}\right)= \\
=\frac{1}{2 \zeta}\left(E^{2}-\mathfrak{B}^{2}-\epsilon^{2}+\beta^{2}+V_{0}^{2}-V^{2}-U_{0}^{2}+U^{2}\right),
\end{gathered}
$$




$$
L_{i}=-j_{e}^{\alpha} X_{\alpha}+j_{m}^{\alpha} Y_{\alpha}
$$

if we consider the potentials $X^{\mu}$ and $Y^{\mu}$ as independent variables. The interaction Lagrangian $L_{i}$ describes the minimal interaction of the vector and pseudovector bosons $X^{\mu}, Y^{\mu}$ with the currents $j_{e}^{\alpha}, j_{m}^{\alpha}$ that produce them. Even if these currents are not conserved the propagators of bosons $X^{\mu}$ and $Y^{\mu}$ have the form (8).

\section{Results}

Use of equations (5), (11), (14) instead of the Proca equations allows describing the intermediate massive bosons in a renormalized way even if they are produced by non-conserved currents. The non-conserved currents are met in the study of the weak interaction. Let us use an example of the weak interaction of the charged currents in order to show the usefulness of the Eqs. (5), (11), (14) to describe the vector and pseudovector bosons.

In the Weinberg-Salam electroweak model the charged current interaction is given by the Lagrangian [8]:

$$
L_{i}=-\frac{g c}{2 \sqrt{2}} \bar{e} \gamma^{\mu}\left(1-\gamma^{5}\right) W_{\mu}^{-} \nu-\frac{g c}{2 \sqrt{2}} \bar{\nu} \gamma^{\mu}\left(1-\gamma^{5}\right) W_{\mu}^{+} e .
$$

Here, $\gamma^{5}=i \gamma^{0} \gamma^{1} \gamma^{2} \gamma^{3}$. The potentials $W_{\mu}^{+}, W_{\mu}^{-}$describe the charged bosons. Let us show that the Lagrangian (30) coincides with (29), if we choose as the currents $j_{e}^{\mu}$, $j_{m}^{\mu}$ the following combinations in Eq. (29):

$$
\begin{gathered}
j_{e}^{\mu}=\frac{g c}{\sqrt{2}}\left(\bar{\nu} \gamma^{\mu} e+\bar{e} \gamma^{\mu} \nu\right), \\
j_{m}^{\mu}=i \frac{g c}{\sqrt{2}}\left(\bar{\nu} \gamma^{\mu} \gamma^{5} e-\bar{e} \gamma^{\mu} \gamma^{5} \nu\right) .
\end{gathered}
$$

Really, the Lagrangian (29) for the currents (31)-(32) is the following:

$$
\begin{gathered}
L_{i}=-j_{e}^{\mu} X_{\mu}+j_{m}^{\mu} Y_{\mu}= \\
-\frac{g c}{\sqrt{2}}\left[\left(\bar{e} \gamma^{\mu} \nu+\bar{\nu} \gamma^{\mu} e\right) X_{\mu}+i\left(\bar{e} \gamma^{\mu} \gamma^{5} \nu-\bar{\nu} \gamma^{\mu} \gamma^{5} e\right) Y_{\mu}\right]= \\
-\frac{g c}{\sqrt{2}}\left[\bar{e} \gamma^{\mu}\left(X_{\mu}+i \gamma^{5} Y_{\mu}\right) \nu+\bar{\nu} \gamma^{\mu}\left(X_{\mu}-i \gamma^{5} Y_{\mu}\right) e\right]= \\
-\frac{g c}{2 \sqrt{2}} \bar{e} \gamma^{\mu}\left[\left(1+\gamma^{5}\right)+\left(1-\gamma^{5}\right)\right]\left(X_{\mu}+i \gamma^{5} Y_{\mu}\right) \nu- \\
-\frac{g c}{2 \sqrt{2}} \bar{\nu} \gamma^{\mu}\left[\left(1+\gamma^{5}\right)+\left(1-\gamma^{5}\right)\right]\left(X_{\mu}-i \gamma^{5} Y_{\mu}\right) e= \\
-\frac{g c}{2 \sqrt{2}} \bar{e} \gamma^{\mu}\left[\left(1+\gamma^{5}\right)\left(X_{\mu}+i Y_{\mu}\right)+\left(1-\gamma^{5}\right)\left(X_{\mu}-i Y_{\mu}\right)\right] \nu- \\
-\frac{g c}{2 \sqrt{2}} \bar{\nu} \gamma^{\mu}\left[\left(1+\gamma^{5}\right)\left(X_{\mu}-i Y_{\mu}\right)+\left(1-\gamma^{5}\right)\left(X_{\mu}+i Y_{\mu}\right)\right] e .
\end{gathered}
$$

Due to the fact that only the left neutrino exists, all terms with the factor $1+\gamma^{5}$ are zeros, and Eq. (33) becomes:

$$
L_{i}=-\frac{g c}{2 \sqrt{2}} \bar{e} \gamma^{\mu}\left(1-\gamma^{5}\right) W_{\mu}^{-} \nu-\frac{g c}{2 \sqrt{2}} \bar{\nu} \gamma^{\mu}\left(1-\gamma^{5}\right) W_{\mu}^{+} e .
$$

In this expression

$$
W_{\mu}^{-}=X_{\mu}-i Y_{\mu}, \quad W_{\mu}^{+}=X_{\mu}+i Y_{\mu} .
$$

The Lagrangian (34) coincides with (30). In such context, we must conclude that charged current interaction is $\mathrm{V}$-A in its' nature, because only left neutrino exists. The potentials $W_{\mu}^{+}$and $W_{\mu}^{-}$are given by the combination of the Eqs. (7) and (13) :

$$
\square_{W^{-\mu}}^{W^{+\mu}}+\varkappa^{2}{ }_{W^{-\mu}}^{W^{+\mu}}=\begin{aligned}
& \zeta\left(j_{e}^{\mu}+i j_{m}^{\mu}\right)=\zeta \frac{g c}{\sqrt{2}} \bar{\nu} \gamma^{\mu}\left(1-\gamma^{5}\right) e \\
& \zeta\left(j_{e}^{\mu}-i j_{m}^{\mu}\right)=\zeta \frac{g c}{\sqrt{2}} \bar{e} \gamma^{\mu}\left(1-\gamma^{5}\right) \nu
\end{aligned} .
$$

We can introduce fields:

$$
\begin{gathered}
\boldsymbol{E}^{ \pm}=-\frac{1}{c} \frac{\partial \boldsymbol{W}^{ \pm}}{\partial t}-\nabla W_{0}^{ \pm} \mp i \nabla \times \boldsymbol{W}^{ \pm}, \\
\epsilon^{ \pm}=-\frac{1}{c} \frac{\partial W_{0}^{ \pm}}{\partial t}+\nabla \cdot \boldsymbol{W}^{ \pm}, \quad V_{\mu}^{ \pm}=-\varkappa W_{\mu}^{ \pm} .
\end{gathered}
$$

These fields are determined from the equations

$$
\begin{gathered}
\frac{1}{c} \frac{\partial \epsilon^{ \pm}}{\partial t}+\nabla \cdot \boldsymbol{E}^{ \pm}-\varkappa V_{0}^{ \pm}=\zeta c \rho^{ \pm}, \\
\frac{1}{c} \frac{\partial \boldsymbol{E}^{ \pm}}{\partial t} \mp i \nabla \times \boldsymbol{E}^{ \pm}+\nabla \epsilon^{ \pm}+\varkappa \boldsymbol{V}^{ \pm}=-\zeta \boldsymbol{j}^{ \pm},
\end{gathered}
$$

where $\rho^{ \pm}=\rho_{e} \pm i \rho_{m}, \boldsymbol{j}^{ \pm}=\boldsymbol{j}_{e} \pm i \boldsymbol{j}_{m}$. As can be seen the superpositions of the vector and pseudovector currents $j_{e}^{\mu} \pm i j_{m}^{\mu}$ create the potentials $W^{+\mu}$ and $W^{-\mu}$. Each superposition creates those potential, with which it interacts in Eq. (34). Though the currents (31) and (32) are not conserved, the propagators of the bosons $W^{+\mu}$ and $W^{-\mu}$ still have the same form (8).

It is also follows from Eq. (35) that the charged bosons can be considered as the superpositions of two states. These states are determined by the modified Maxwell's equations (15)-(18) with the currents (31) and (32). One of them is described by the vector potential $X^{\mu}$, the other by the pseudovector potential $Y^{\mu}$. These potentials interact minimally with the currents (31) and (32), respectively.

\section{Discussion}

Thus, massive intermediate bosons can be given a renormalized description with no reference to local calibration symmetry and spontaneous symmetry breaking. Non-renormalizability is not connected to the boson massiveness but with inappropriate application of the Proca equations to the non-conserved currents. Nonconserved currents have to be described by equations (5), (11), (14) instead of the Proca equations. The bosons, the leptons and the weak interaction of their charged currents can be described by the Lagrangian

$$
L=L_{b}+\hbar c^{2} L_{l}+L_{i}
$$

where

$$
\begin{aligned}
L_{l}= & \frac{i}{2}\left(\bar{e} \gamma^{\mu} \frac{\partial e}{\partial x^{\mu}}-\frac{\partial \bar{e}}{\partial x^{\mu}} \gamma^{\mu} e\right)-\frac{m_{e} c}{\hbar} \bar{e} e+ \\
& +\frac{i}{2}\left(\bar{\nu} \gamma^{\mu} \frac{\partial \nu}{\partial x^{\mu}}-\frac{\partial \bar{\nu}}{\partial x^{\mu}} \gamma^{\mu} \nu\right) .
\end{aligned}
$$

It should be pointed out that the interaction Lagrangian in (41) can be used as (29), which is similar to the electrodynamics interaction Lagrangian. The boson propagator is different from the photon propagator by the 
mass term in the denominator only. That is why such interaction is renormalized. Because the Lagrangians (29) and (30) coincide, the results obtained with the Lagrangian (29) and obtained with the Weinberg-Salam model Lagrangian (30) coincide also.

We do not touch on here other aspects of the weak interaction, as the aim of the article is to show the usefulness of use of the equations (5), (11), (14) instead of the Proca equation for the description of the vector and pseudovector bosons in the case of non-conserved current interactions.

\section{Acknowledgements}

The author would like to thank Prof. Lev B.I., Prof. Lukyanets S.P. and Prof. Zhmudskyy O.O. for stimulating discussions.

\section{REFERENCES}

[1] S. Weinberg, A Model of Leptons, Phys. Rev. Lett., 19, 1264 - 1266, (1967).

[2] A. Salam, Elementary Particle Theory: Relativistic Groups and Analyticity, N.Svartholm, Almquist and
Wiksell, Stockhom, Nobel symposium 8 (1968).

[3] S. I. Kruglov, Symmetry and Electromagnetic Interaction of Fields with Multi-Spin (Nova Science Publishers, Huntington, New York, 2001).

[4] S. I. Kruglov, Bosonic Fields with Two Mass and Spin States, Int. J. Mod. Phys. A16, 4925-4938 (2001).

[5] S. I. Kruglov, Internal Symmetry Group and Density Matrix of Fields with Spins 0, 1. Hadronic J. 24, 167-185, (2001).

[6] S. I. Kruglov, On Torsion Fields in Higher Derivative Quantum Gravity, Annales Fond. Broglie 32, no 2-3, 323-334, (2007).

[7] N. Cabibbo and E. Ferrari, Quantum electrodynamics with Dirac monopoles, Nuovo Cimento, 23, 1147 1154, (1962).

[8] E. D. Commins and P. H. Bucksbaum, Weak interactions of leptons and quarks, Cambridge University Press, Cambridge, 1983). 